\title{
ON THE EXISTENCE OF POSITIVE SOLUTIONS OF NONLINEAR SECOND ORDER DIFFERENTIAL EQUATIONS
}

WEI-CHENG LIAN, FU-HSIANG WONG, AND CHEH-CHIH YEH

(Communicated by Hal L. Smith )

Dedicated to Professor Taro Yoshizawa on his 75th birthday

Abstract. Under suitable conditions on $f(t, u)$, the boundary value problem

$(\mathrm{BVP}) \quad\left\{\begin{array}{l}(E) u^{\prime \prime}(t)+f(t, u(t))=0 \text { in }(0,1), \\ (B C)\left\{\begin{array}{l}\alpha u(0)-\beta u^{\prime}(0)=0, \\ \gamma u(1)+\delta u^{\prime}(1)=0\end{array}\right.\end{array}\right.$

has at least one positive solution. Moreover, we also apply this main result to establish several existence theorems of multiple positive solutions for some nonlinear (elliptic) differential equations.

\section{INTRODUCTION}

There has recently been an increased interest in studying the existence of positive solutions of the following boundary value problem

$(\mathrm{BVP})$

$$
\left\{\begin{array}{c}
(E) u^{\prime \prime}(t)+f(t, u(t))=0 \text { in }(0,1), \\
(B C)\left\{\begin{array}{l}
\alpha u(0)-\beta u^{\prime}(0)=0 \\
\gamma u(1)+\delta u^{\prime}(1)=0
\end{array}\right.
\end{array}\right.
$$

in the last fifteen to twenty-five years; see, for example, Bandle, Coffman and Marcus [1], Bandle and Kwong [2], Coffman and Marcus [3], H. Dang and K. Schmit [4], Erbe [6], Erbe, Hu and Wang [7], Erbe and Wang [8], Garaizar [9], Iffland [11], Santanilla [13], Wang [14] and Wong [15].

In 1994, Erbe, Hu and Wang $[7,8]$ showed the following excellent results:

Theorem A (Erbe and Wang [8]). Suppose that

(A.1) $f \in C([0,1] \times[0, \infty) ;[0, \infty))$,

(A.2) $\alpha, \beta, \gamma, \delta \geq 0$ and $\rho:=\gamma \beta+\alpha \gamma+\alpha \delta>0$.

Received by the editors September 21, 1994.

1991 Mathematics Subject Classification. Primary 34B15.

Key words and phrases. Boundary value problems, positive solution, operator equation, cone, fixed point. 
Then, $(B V P)$ has at least one positive solution in the case

(i) $\lim _{u \rightarrow 0^{+}} \max _{t \in[0,1]} \frac{f(t, u)}{u}=0$ and $\lim _{u \rightarrow \infty} \min _{t \in[0,1]} \frac{f(t, u)}{u}=\infty$ (superlinear), or

(ii) $\lim _{u \rightarrow 0^{+}} \min _{t \in[0,1]} \frac{f(t, u)}{u}=\infty$ and $\lim _{u \rightarrow \infty} \max _{t \in[0,1]} \frac{f(t, u)}{u}=0$ (sublinear).

Theorem B (Erbe, $\mathrm{Hu}$ and Wang [7]). Assume that $\left(\mathrm{A}_{1}\right),\left(\mathrm{A}_{2}\right)$ and the following assumptions hold:

(A.3) $\lim _{u \rightarrow 0^{+}} \min _{t \in[0,1]} \frac{f(t, u)}{u}=\lim _{u \rightarrow \infty} \min _{t \in[0,1]} \frac{f(t, u)}{u}=\infty$,

(A.4) there is a $p>0$ such that

$$
f(t, u) \leq \eta p \text { on }[0,1] \times[0, p]
$$

where $\eta=\left(\int_{0}^{1} G(s, s) d s\right)^{-1}=\frac{6 \rho}{6 \delta \beta+3 \gamma \beta+\alpha \gamma+3 \alpha \delta}$. Here $G(t, s)$ is the Green's function of

$$
u^{\prime \prime}(t)=0 \text { in }(0,1)
$$

with respect to the boundary value condition $(B C)$.

Then, $(B V P)$ has at least two positive solutions $u_{1}$ and $u_{2}$ such that

$$
0<\left\|u_{1}\right\|<p<\left\|u_{2}\right\|
$$

Theorem C (Erbe, Hu and Wang [7]). Assume that $\left(\mathrm{A}_{1}\right),\left(\mathrm{A}_{2}\right)$ and the following assumptions hold:

(A.5) $\lim _{u \rightarrow 0^{+}} \max _{t \in[0,1]} \frac{f(t, u)}{u}=\lim _{u \rightarrow \infty} \max _{t \in[0,1]} \frac{f(t, u)}{u}=0$,

(A.6) there is a $p>0$ such that

$$
f(t, u) \geq \lambda p \text { on }\left[\frac{1}{4}, \frac{3}{4}\right] \times[\sigma p, p]
$$

where $\lambda=\left(\int_{\frac{1}{4}}^{\frac{3}{4}} G\left(\frac{1}{2}, s\right) d s\right)^{-1}$ and $\sigma=\min \left\{\frac{\gamma+4 \delta}{4(\gamma+\delta)}, \frac{\alpha+4 \beta}{4(\alpha+\beta)}\right\}$.

Then, $(B V P)$ has at least two positive solutions $u_{1}$ and $u_{2}$ such that

$$
0<\left\|u_{1}\right\|<p<\left\|u_{2}\right\|
$$

Let

$$
\begin{aligned}
\max f_{0} & :=\lim _{u \rightarrow 0^{+}} \max _{t \in[0,1]} \frac{f(t, u)}{u}, \\
\min f_{0} & :=\lim _{u \rightarrow 0^{+}} \min _{t \in[0,1]} \frac{f(t, u)}{u}, \\
\max _{\infty} & :=\lim _{u \rightarrow \infty} \max _{t \in[0,1]} \frac{f(t, u)}{u},
\end{aligned}
$$

and

$$
\operatorname{minf} f_{\infty}:=\lim _{u \rightarrow \infty} \min _{t \in[0,1]} \frac{f(t, u)}{u} .
$$

Then, it follows from Theorems A, B and C that

(I) $\max f_{0}=0$ and $\min f_{\infty}=\infty$ implies (BVP) has at least one positive solution,

(II) $\min f_{0}=\infty$ and $\max f_{\infty}=0$ implies (BVP) has at least one positive solution, 
(III)

$$
\left\{\begin{array}{l}
\min f_{0}=\min f_{\infty}=\infty, \\
f(t, u) \leq \eta p \text { on }[0,1] \times[0, p]
\end{array}\right.
$$

implies (BVP) has at least two positive solutions,

(IV)

$$
\left\{\begin{array}{l}
\max f_{0}=\max f_{\infty}=0, \\
f(t, u) \geq \lambda p \text { on }[0,1] \times[\sigma p, p]
\end{array}\right.
$$

implies (BVP) has at least two positive solutions.

Seeing such a fact, we can not but ask "whether or not we can obtain a similar conclusion, if $\max f_{0}, \min f_{0}, \max f_{\infty}, \min f_{\infty} \notin\{0, \infty\} . "$ Inspired by the above-mentioned results, we attempt to establish a simple criterion for the existence of positive solutions of (BVP), which is a generalization of Theorems A, B, and C and gives a positive answer to the question stated above.

\section{MAin Results}

Let $\alpha, \beta, \gamma, \delta \geq 0$ and $\rho:=\gamma \beta+\alpha \gamma+\alpha \delta>0$. In order to abbreviate our discussion, throughout this paper, we suppose that the following assumptions hold: $\left(\mathrm{C}_{1}\right) k(t, s)$ is the Green's function of the differential equation

$$
u^{\prime \prime}(t)=0 \text { in }(0,1)
$$

with respect to the boundary value condition $(B C)$;

$\left(\mathrm{C}_{2}\right) \quad 0<M:=\min \left\{\frac{\gamma+4 \delta}{4(\gamma+\delta)}, \frac{\alpha+4 \beta}{4(\alpha+\beta)}\right\}<1$;

$\left(\mathrm{C}_{3}\right) \quad f \in C([0,1] \times[0, \infty) ;[0, \infty))$.

In order to discuss our main result (Theorem 1 below), we need the following two useful lemmas:

Lemma D. Suppose that $k(t, s)$ is defined as in $\left(\mathrm{C}_{1}\right)$. Then we have the following results:

$$
\left\{\begin{array}{l}
\left(R_{1}\right) \frac{k(t, s)}{k(s, s)} \leq 1, \quad \text { for } t \in[0,1] \text { and } s \in[0,1] \\
\left(R_{2}\right) \frac{k(t, s)}{k(s, s)} \geq M, \quad \text { for } t \in\left[\frac{1}{4}, \frac{3}{4}\right] \text { and } s \in[0,1]
\end{array}\right.
$$

Proof. Let

$$
\varphi(t):=(\gamma+\delta-\gamma t) \text { and } \psi(t):=\beta+\alpha t \text { for } t \in[0,1]
$$

Then,

which implies

$$
k(t, s)=\left\{\begin{array}{l}
\frac{1}{\rho} \varphi(t) \psi(s), 0 \leq s \leq t \leq 1 \\
\frac{1}{\rho} \varphi(s) \psi(t), 0 \leq t \leq s \leq 1
\end{array}\right.
$$

$$
\frac{k(t, s)}{k(s, s)}=\left\{\begin{array}{l}
\frac{\varphi(t)}{\varphi(s)}, 0 \leq s \leq t \leq 1 \\
\frac{\psi(t)}{\psi(s)}, 0 \leq t \leq s \leq 1
\end{array}\right.
$$


Hence, we obtain the following desired results:

$$
\frac{k(t, s)}{k(s, s)} \leq 1 \text { for } t \in[0,1]
$$

and

$$
\frac{k(t, s)}{k(s, s)} \geq\left\{\begin{array}{l}
\frac{\gamma+4 \delta}{4(\gamma+\delta)} \geq M, 0 \leq s \leq t \leq \frac{3}{4} \\
\frac{\alpha+4 \beta}{4(\alpha+\beta)} \geq M, \frac{1}{4} \leq t \leq s \leq 1
\end{array}\right.
$$

Lemma E (Deimling [5] and Krasnoselskii [12]). Let $E$ be a Banach space, and let $K \subset E$ be a cone in $E$. Assume $\Omega_{1}, \Omega_{2}$ are open subsets of $E$ with $0 \in \Omega_{1}, \overline{\Omega_{1}} \subset \Omega_{2}$, and let

$$
A: K \cap\left(\overline{\Omega_{2}} \backslash \Omega_{1}\right) \longrightarrow K
$$

be a completely continuous operator such that either

(i) $\|A u\| \leq\|u\|, u \in K \cap \partial \Omega_{1}$ and $\|A u\| \geq\|u\|, u \in K \cap \partial \Omega_{2}$; or

(ii) $\|A u\| \geq\|u\|, u \in K \cap \partial \Omega_{1}$ and $\|A u\| \leq\|u\|, u \in K \cap \partial \Omega_{2}$.

Then $A$ has a fixed point in $K \cap\left(\overline{\Omega_{2}} \backslash \Omega_{1}\right)$.

Now, we can state and prove our main result.

Theorem 1. Assume that there exist two distinct positive constants $\lambda, \eta$ such that

$$
f(t, u) \leq \lambda\left(\int_{0}^{1} k(s, s) d s\right)^{-1} \text { on }[0,1] \times[0, \lambda]
$$

and

$$
f(t, u) \geq \eta\left(\int_{\frac{1}{4}}^{\frac{3}{4}} k\left(\frac{1}{2}, s\right) d s\right)^{-1} \text { on }\left[\frac{1}{4}, \frac{3}{4}\right] \times[M \eta, \eta] .
$$

Then $(B V P)$ has at least one positive solution $u$ such that $\|u\|$ between $\lambda$ and $\eta$, where $\|u\|:=\sup _{t \in[0,1]}|u(t)|$.

Proof. Without loss of generality, we may assume that $\lambda<\eta$. It is clear that $(B V P)$ has a solution $u=u(t)$ if, and only if, $u$ is the solution of the operator equation

$$
u(t)=\int_{0}^{1} k(t, s) f(s, u(s)) d s:=A u(t), u \in C[0,1] .
$$

Let $K$ be a cone in $C[0,1]$ given by

$$
K=\left\{u \in C[0,1] \mid u(t) \geq 0, \min _{t \in\left[\frac{1}{4}, \frac{3}{4}\right]} u(t) \geq M\|u\|\right\} .
$$

It follows from the definition of $K$ and Lemma $D$ that

$$
\begin{aligned}
\min _{t \in\left[\frac{1}{4}, \frac{3}{4}\right]}(A u)(t) & =\min _{t \in\left[\frac{1}{4}, \frac{3}{4}\right]} \int_{0}^{1} k(t, s) f(s, u(s)) d s \\
& \geq M \int_{0}^{1} k(s, s) f(s, u(s)) d s\left(u \operatorname{sing}\left(R_{2}\right)\right) \\
& \geq M \int_{0}^{1} k(t, s) f(s, u(s)) d s\left(u \operatorname{sing}\left(R_{1}\right)\right) .
\end{aligned}
$$


Hence, $\min _{t \in\left[\frac{1}{4}, \frac{3}{4}\right]}(A u)(t) \geq M\|A u\|$, which implies $A K \subset K$. Furthermore, it is easy to check that $A: K \rightarrow K$ is completely continuous. In order to complete the proof, we separate the rest of the proof into the following two steps:

Step (I) Let $\Omega_{1}:=\{u \in K\|\| u \|<\lambda\}$. It follows from (2) and Lemma D that for $u \in \partial \Omega_{1}$,

$$
\begin{aligned}
(A u)(t) & =\int_{0}^{1} k(t, s) f(s, u(s)) d s \\
& \leq \int_{0}^{1} k(s, s) f(s, u(s)) d s \\
& \leq \lambda\left(\int_{0}^{1} k(s, s) d s\right)^{-1}\left(\int_{0}^{1} k(s, s) d s\right) \frac{\|u\|}{\lambda} \\
& =\|u\| .
\end{aligned}
$$

Hence,

$$
\|A u\| \leq\|u\| \text { for } u \in \partial \Omega_{1} .
$$

Step (II) Let $\Omega_{2}:=\{u \in K \mid\|u\|<\eta\}$. It follows from the definitions of $\|u\|$ and K that

$$
\left\{\begin{array}{l}
u(t) \leq\|u\|=\eta \text { for } t \in[0,1], \\
u(t) \geq \min _{t \in\left[\frac{1}{4}, \frac{3}{4}\right]} u(t) \geq M\|u\|=M \eta \text { for } t \in\left[\frac{1}{4}, \frac{3}{4}\right],
\end{array}\right.
$$

for $u \in \partial \Omega_{2}$, which implies

$$
M \eta \leq u(t) \leq \eta \text { for } t \in\left[\frac{1}{4}, \frac{3}{4}\right]
$$

Hence, by (3),

$$
\begin{aligned}
(A u)\left(\frac{1}{2}\right) & =\int_{0}^{1} k\left(\frac{1}{2}, s\right) f(s, u(s)) d s \\
& \geq \int_{\frac{1}{4}}^{\frac{3}{4}} k\left(\frac{1}{2}, s\right) f(s, u(s)) d s \\
& \geq \eta\left(\int_{\frac{1}{4}}^{\frac{3}{4}} k\left(\frac{1}{2}, s\right) d s\right)^{-1}\left(\int_{\frac{1}{4}}^{\frac{3}{4}} k\left(\frac{1}{2}, s\right) d s\right) \frac{\|u\|}{\eta} \\
& =\|u\| .
\end{aligned}
$$

Thus,

$$
\|A u\| \geq\|u\| \text { for } u \in \partial \Omega_{2} .
$$

Therefore, by the first part of Lemma E, we complete the proof.

Remark 2. Since

$$
\left(\int_{0}^{1} k(s, s) d s\right)^{-1}:=A=\frac{6 \rho}{6 \delta \beta+3 \gamma \beta+\alpha \gamma+3 \alpha \delta},
$$

and

$$
\left(\int_{\frac{1}{4}}^{\frac{3}{4}} k\left(\frac{1}{2}, s\right) d s\right)^{-1}:=B=\frac{8 \rho}{4 \beta \delta+2 \beta \gamma+\alpha \gamma+2 \alpha \delta},
$$


then, we have the following results:

(a) Suppose that $\max f_{0}:=C_{1} \in[0, A)$. Taking $\epsilon=A-C_{1}>0$, there exists $\lambda_{1}>0\left(\lambda_{1}\right.$ can be chosen small arbitrarily) such that

$$
\max _{t \in[0,1]} \frac{f(t, u)}{u} \leq \epsilon+C_{1}=A \text { on }\left(0, \lambda_{1}\right] \text {. }
$$

Hence,

$$
f(t, u) \leq A u \leq A \lambda_{1} \text { on }[0,1] \times\left[0, \lambda_{1}\right],
$$

which satisfies the hypothesis (2) of Theorem 1.

(b) Suppose that $\min f_{\infty}:=C_{2} \in\left(\frac{B}{M}, \infty\right]$. Taking $\epsilon=C_{2}-\frac{B}{M}>0$, there exists $\eta_{1}>0\left(\eta_{1}\right.$ can be chosen large arbitrarily) such that

$$
\min _{t \in[0,1]} \frac{f(t, u)}{u} \geq-\epsilon+C_{2}=\frac{B}{M} \text { on }\left[M \eta_{1}, \infty\right) .
$$

Hence,

$$
f(t, u) \geq \frac{B}{M} u \geq \frac{B}{M} M \eta_{1}=B \eta_{1} \text { on }\left[\frac{1}{4}, \frac{3}{4}\right] \times\left[M \eta_{1}, \eta_{1}\right] \subseteq[0,1] \times\left[M \eta_{1}, \infty\right),
$$

which satisfies the hypothesis (3) of Theorem 1.

(c) Suppose that $\min f_{0}:=C_{3} \in\left(\frac{B}{M}, \infty\right]$. Taking $\epsilon=C_{3}-\frac{B}{M}>0$, there exists $\eta_{2}>0\left(\eta_{2}\right.$ can be chosen small arbitrarily) such that

$$
\min _{t \in[0,1]} \frac{f(t, u)}{u} \geq-\epsilon+C_{3}=\frac{B}{M} \text { on }\left(0, \eta_{2}\right] .
$$

Hence,

$$
f(t, u) \geq \frac{B}{M} u \geq \frac{B}{M} M \eta_{2}=B \eta_{2} \text { on }\left[\frac{1}{4}, \frac{3}{4}\right] \times\left[M \eta_{2}, \eta_{2}\right] \subseteq[0,1] \times\left[0, \eta_{2}\right],
$$

which satisfies the hypothesis (3) of Theorem 1.

(d) Suppose that $\max _{\infty}:=C_{4} \in[0, A)$. Taking $\epsilon=A-C_{4}>0$, there exists $\delta>0$ ( $\delta$ can be chosen large arbitrarily) such that

$$
\max _{t \in[0,1]} \frac{f(t, u)}{u} \leq \epsilon+C_{4}=A \text { on }[\delta, \infty) .
$$

Hence, we have the following two cases:

Case (I). Assume that $\max _{t \in[0,1]} f(t, u)$ is bounded, say,

$$
f(t, u) \leq L \text { on }[0,1] \times[0, \infty) .
$$

Taking $\lambda_{2}=\frac{L}{A}$ (since $\mathrm{L}$ can be chosen large arbitrarily, $\lambda_{2}$ can be chosen large arbitrarily, too),

$$
f(t, u) \leq L=A \lambda_{2} \text { on }[0,1] \times\left[0, \lambda_{2}\right] \subseteq[0,1] \times[0, \infty) .
$$

Case (II). Assume that $\max _{t \in[0,1]} f(t, u)$ is unbounded, hence, there exists $\lambda_{2} \geq \delta\left(\lambda_{2}\right.$ can be chosen large arbitrarily) and $t_{0} \in[0,1]$ such that

$$
f(t, u) \leq f\left(t_{0}, \lambda_{2}\right) \text { on }[0,1] \times\left[0, \lambda_{2}\right]
$$

It follows from $\lambda_{2} \geq \delta$ and (4) that

$$
f(t, u) \leq f\left(t_{0}, \lambda_{2}\right) \leq A \lambda_{2} \text { on }[0,1] \times\left[0, \lambda_{2}\right] .
$$

By Cases (I) and (II), the hypothesis (2) of Theorem 1 is satisfied.

It follows from Remark 2 that the following corollaries hold. They are generalizations of Theorems A, B and C, respectively. 
Corollary 3. Let $A$ and $B$ be defined as in Remark 2. Then, $(B V P)$ has at least one positive solution in the case

(1) $\max f_{0}=C_{1} \in[0, A)$ and $\min f_{\infty}=C_{2} \in\left(\frac{B}{M}, \infty\right]$, or

(2) $\min f_{0}=C_{3} \in\left(\frac{B}{M}, \infty\right]$ and $\max f_{\infty}=C_{4} \in[0, A)$.

Proof. It follows from Remark 2 and Theorem 1 that the desired result holds, immediately.

Corollary 4. Let $A$ and $B$ be defined as in Remark 2. Then, $(B V P)$ has at least two positive solutions $u_{1}$ and $u_{2}$ such that

$$
0<\left\|u_{1}\right\|<\lambda^{*}<\left\|u_{2}\right\|
$$

if the following hypotheses hold:

$\left(\mathrm{H}_{1}\right) \min f_{\infty}=C_{2}, \min f_{0}=C_{3} \in\left(\frac{B}{M}, \infty\right]$,

$\left(\mathrm{H}_{2}\right)$ there exists $\lambda^{*}>0$ such that

$$
f(t, u) \leq A \lambda^{*} \text { on }[0,1] \times\left[0, \lambda^{*}\right] .
$$

Proof. It follows from Remark 2 that there exist two real numbers $\eta_{1}$ and $\eta_{2}$ satisfying

$$
\begin{gathered}
0<\eta_{2}<\lambda^{*}<\eta_{1}, \\
f(t, u) \geq B \eta_{1} \text { on }\left[\frac{1}{4}, \frac{3}{4}\right] \times\left[M \eta_{1}, \eta_{1}\right],
\end{gathered}
$$

and

$$
f(t, u) \geq B \eta_{2} \text { on }\left[\frac{1}{4}, \frac{3}{4}\right] \times\left[M \eta_{2}, \eta_{2}\right] .
$$

Hence, by Theorem 1, we see that (BVP) has two positive solutions $u_{1}$ and $u_{2}$ such that

$$
\eta_{2}<\left\|u_{1}\right\|<\lambda^{*}<\left\|u_{2}\right\|<\eta_{1} .
$$

Thus, we complete the proof.

Corollary 5. Let $A$ and $B$ be defined as in Remark 2. Then, $(B V P)$ has at least two positive solutions $u_{1}$ and $u_{2}$ such that

$$
0<\left\|u_{1}\right\|<\eta^{*}<\left\|u_{2}\right\|
$$

if the following hypotheses hold:

$\left(\mathrm{H}_{3}\right) \max f_{0}=C_{1}, \max f_{\infty}=C_{4} \in[0, A)$,

$\left(\mathrm{H}_{4}\right)$ there exists $\eta^{*}>0$ such that

$$
f(t, u) \geq B \eta^{*} \text { on }\left[\frac{1}{4}, \frac{3}{4}\right] \times\left[M \eta^{*}, \eta^{*}\right] .
$$

Proof. It follows from Remark 2 that there exist two real numbers $\lambda_{1}$ and $\lambda_{2}$ satisfying

$$
\begin{gathered}
0<\lambda_{1}<\eta^{*}<\lambda_{2}, \\
f(t, u) \leq A \lambda_{1} \text { on }[0,1] \times\left[0, \lambda_{1}\right],
\end{gathered}
$$




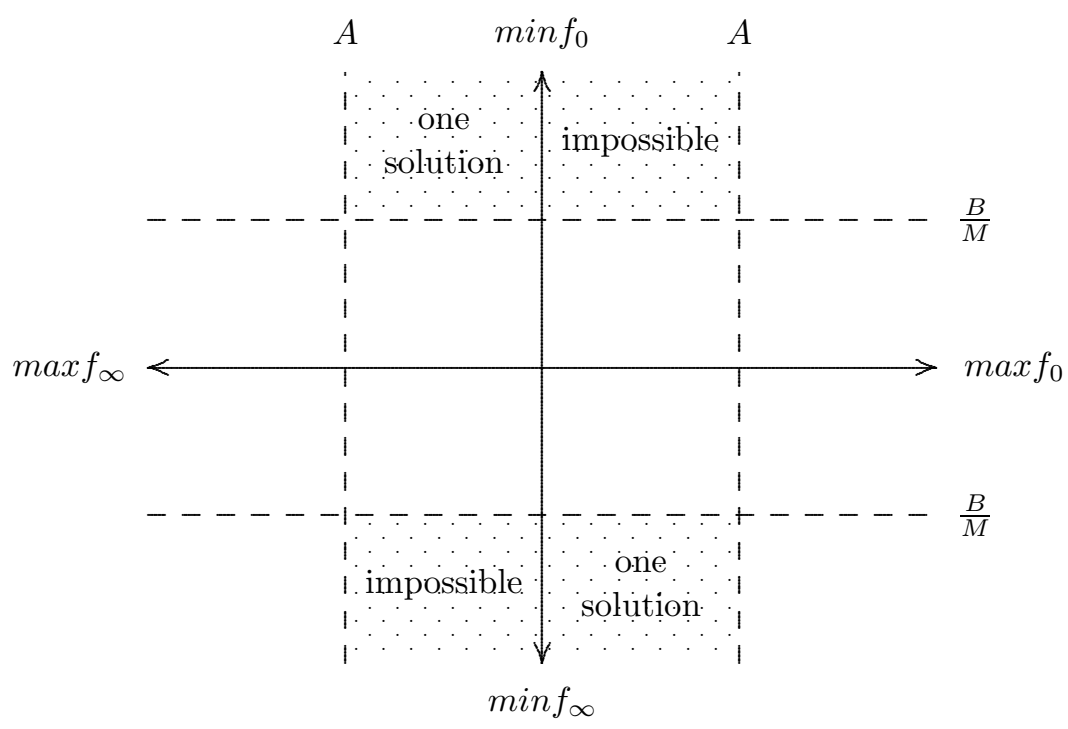

FIGURE 2.1

and

$$
f(t, u) \leq A \lambda_{2} \text { on }[0,1] \times\left[0, \lambda_{2}\right]
$$

Hence, by Theorem 1, we see that (BVP) has two positive solutions $u_{1}$ and $u_{2}$ such that

$$
\lambda_{1}<\left\|u_{1}\right\|<\eta^{*}<\left\|u_{2}\right\|<\lambda_{2}
$$

Thus, we complete the proof.

Remark 6. There are many functions $f(t, u)$ which do not satisfy " $\max f_{0}, \min f_{0}$, $\max f_{\infty}, \min f_{\infty} \in\{0, \infty\} "$, for example, $f(t, u):=\frac{e^{u}-1}{1+t^{2}}\left(\max f_{0}=1\right.$ and $\min f_{0}=$ $\left.\frac{1}{2}\right), f(t, u):=(t+1) \sinh u\left(\max f_{0}=2\right.$ and $\left.\min f_{0}=1\right), f(t, u):=u+t^{2} e^{-u}\left(\max f_{0}\right.$ $\left.=\infty, \min f_{0}=\max f_{\infty}=\min f_{\infty}=1\right)$.

Remark 7. It follows from Corollaries 3, 4, 5 that we obtain Figure 2.1.

Remark 8. If we consider the radial solutions to the boundary value problem of the following semilinear elliptic equation of the form

$\left(\right.$ BVP.1) $\left\{\begin{array}{l}\Delta u+g(|x|) f(u)=0,0<R_{1}<|x|<R_{2}, \quad N \geq 2, \\ (B C .1)\left\{\begin{array}{l}\alpha u(x)-\beta \frac{\partial u}{\partial n}(x)=0,|x|=R_{1}, \\ \gamma u(x)-\delta \frac{\partial u}{\partial n}(x)=0,|x|=R_{2},\end{array}\right.\end{array}\right.$ 
then (BVP .1) can be reduced to the following boundary problem:

(BVP.2)

$$
\left\{\begin{array}{l}
u^{\prime \prime}(r)+\frac{N-1}{r} u^{\prime}(r)+g(r) f(u(r))=0,0<R_{1}<r<R_{2}, \\
(B C .2)\left\{\begin{array}{l}
\alpha u\left(R_{1}\right)-\beta \frac{\partial u}{\partial n}\left(R_{1}\right)=0, \\
\gamma u\left(R_{2}\right)-\delta \frac{\partial u}{\partial n}\left(R_{2}\right)=0 .
\end{array}\right.
\end{array}\right.
$$

Setting $s=-\int_{r}^{R_{2}}\left(1 / t^{N-1}\right) d t, v(s)=u(r(s)), m=-\int_{R_{1}}^{R_{2}}\left(1 / t^{N-1}\right) d t$, (BVP .2) is equivalent to

(BVP.3)

$$
\left\{\begin{array}{l}
v^{\prime \prime}(s)+r^{2(N-1)} g(r(s)) f(v(s))=0, m<s<0 \\
(B C .3)\left\{\begin{array}{l}
\alpha v(m)+\beta R_{1}{ }^{1-N} v^{\prime}(m)=0 \\
\gamma v(0)-\delta R_{2}{ }^{1-N} v^{\prime}(0)=0 .
\end{array}\right.
\end{array}\right.
$$

Furthermore, if $t=\frac{(m-s)}{m}, z(t)=v(s)$, then (BVP .3) can be transformed into

$$
\left\{\begin{array}{l}
z^{\prime \prime}(t)+m^{2} r^{2(N-1)}[m(1-t)] g[r(m(1-t))] f(z(t))=0,0<t<1, \\
(B C .4)\left\{\begin{array}{l}
\alpha z(0)-\beta \frac{R_{1}{ }^{1-N}}{m} z^{\prime}(0)=0, \\
\gamma z(1)+\delta \frac{R_{1}{ }^{1-N}}{m} z^{\prime}(1)=0 .
\end{array}\right.
\end{array}\right.
$$

Hence, we can apply Theorem 1 and Corollaries 3, 4, 5 to (BVP .4), and thereby study the existence of positive radial solutions for (BVP.1).

\section{REFERENCES}

1. C. Bandle, C. V. Coffman and M. Marcus, Nonlinear elliptic problems in annular domains, J. Differential Equations 69 (1987), 322-345. MR 89a:35082

2. C. Bandle and M.K. Kwong, Semilinear elliptic problems in annular domains, J. Appl. Math. Phys. 40 (1989), 245-257. MR 90m:35062

3. C. V. Coffman and M. Marcus, Existence and uniqueness results for semilinear Dirichlet problems in Annuli, Arch. Rational Mech. Anal. 108 (1989), 293-307. MR 90m:35064

4. H. Dang and K. Schmit, Existence of positive solutions for semiliear elliptic equations in annular domain, Diff. and Integ. Equs. 7 (1994), 747-758. MR 94m:35020

5. K. Deimling, Nonlinear functional analysis, Springer, New York, 1985. MR 86j:47001

6. L. H. Erbe, Boundary value problems for ordinary differential equations, Rocky Mountain J. Math. 1 (1970), 709-729. MR 44:4279

7. L. H. Erbe, S. Hu and H. Wang, Multiple positive solutions of some boundary value problems, J. Math. Anal. Appl. 184 (1994), 640-648. MR 95f:34023

8. L. H. Erbe and H. Wang, On the existence of positive solutions of ordinary differential equations, Proc. Amer. Math. Soc. 120 (1994), 743-748. MR 94e:34025

9. X. Garaizar, Existence of positive radial solutions for semiliear elliptic equations in the annulus, J. Differential Equations 70 (1987), 69-72. MR 89f:35019

10. G. B. Gustafson and K. Schmit, Nonzero solutions of boundary value problems for second order ordinary and delay-differential equations, J. Differential Equations 12 (1972), 127-147. MR 49:10959

11. G. Iffland, Positive solutions of a problem of Emden-Fowler type with a free boundary, SIAM J. Math. Anal. 18 (1987), 283-292. MR 88c:34025 
12. M. A. Krasnoselskii, Positive solutions of operator equations, Noordhoff, Groningen, 1964. MR 31:6107

13. J. Santanilla, Nonnegative solutions to boundary value problems for nonlinear first and second order ordinary differential equations, J. Math. Anal. Appl. 126 (1987), 397-408. MR 88h:34014

14. H. Wang, On the existence of positive solutions for semilinear elliptic equations in the annulus, J. Differential Equations 109 (1994), 1-7. MR 95c:35093

15. F. H. Wong, Existence of positive solutions of singular boundary value problems, Nonlinear Analysis T.M.\& A. 21 (1993), 397-406. MR 94i:34056

(W. C. Lian and C. C. Yeh) Department of Mathematics, National Central University, Chung-Li, 32054 Taiwan, Republic of China

E-mail address: Yeh@wangwei.math.ncu.edu.tw

(F. H. Wong) Department of Mathematics and Science, National Taipei Teacher's College, 134, Ho Ping E. RD. Sec. 2, Taipei 10659, Taiwan, Republic of China 